\title{
The optimisation and comparison of re-entry assessment methodologies for use in seismically active mines
}

\author{
SR Tierney Curtin University, Australia \\ IG Morkel Australian Centre for Geomechanics and The University of Western Australia, Australia
}

\begin{abstract}
The hazard posed from large seismic events is often high enough to warrant the exclusion or evacuation of personnel from underground workings. A period of exclusion is often determined following blasts or large events due to the increased risk. The period of exclusion until re-entry occurs is a decision for site geotechnical engineers and mine management that must balance the potential risk to personnel with lost production time and associated costs. There is currently no widely accepted method for determining re-entry times and mine sites typically develop their own rules for exclusions after blasts and large events. A systematic and evidence based approach to the development of re-entry protocols could potentially reduce the risk to personnel from an early re-entry or reduce the lost production from an unnecessary exclusion. Four methods of re-entry assessment have been considered in this paper. The seismic responses at three mines have been modelled and used to optimise each assessment method and gauge the relative success through back-analysis. These same techniques are available for other mines to review their own data and potentially improve their current re-entry protocols. The results of this research indicate that a real-time re-entry assessment method can offer improved outcomes compared to blanket re-entry rules by reducing the average exclusion time while still capturing the same number of large events. The incorporation of event size in the assessment can result in better results than the event count. Vallejos and McKinnon (2009) developed a probabilistic framework for re-entry assessment but this method was found to be less efficient than the blanket rule in the majority of cases in this study. The method would also result in more administration and uncertainty for mine planning and scheduling. Several potential improvements to the analysis techniques, and avenues for further research, have been discussed.
\end{abstract}

Keywords: seismic re-entry, seismic risk, mine seismology

\section{Introduction}

The hazard related to mining-induced seismicity has been reported for well over 100 years in hard rock mines in many countries including: Australia, South Africa and Canada (Potvin \& Hudyma 2001). Research into mine seismicity aims to provide a thorough understanding of the seismic phenomena and a range of risk management strategies (Cook 1976).

The most effective way of minimising the hazard associated with mining seismicity is by employing favourable mine layouts and sequences with a thorough understanding of the physical mechanisms (Potvin 2009). However, since seismicity commonly presents later in a mine's life, there may not be the opportunity to modify the mining method, sequence or layout (Alcott et al. 1998) and, therefore, alternate control systems need to be considered.

Ground support is the main tool employed to control deformation of the rock mass and prevent ejection of material into working areas. Dynamic support systems are often installed but the design is usually based on experience and perceived performance rather than on an extensive understanding of the rock mass mechanisms and support system interactions (Hadjigeorgiou \& Potvin 2011).

The uncertainty in the stability of excavation leads some mines to exclude or evacuate personnel from areas when there is an elevated risk of a large seismic event. Personnel are only allowed to re-enter when 
the risk has dropped to a threshold level. This decision is one of the most critical for onsite engineers to make. Mines often rely on decision-making based on rules of thumb, experience or common sense but this type of judgement can often lead to severe and systematic errors (Kahneman 2003).

Mendecki (2008) argues that there is frequently a motivational bias in human judgement that can lead to poor decisions, and experience based judgements may not be readily applied when mining conditions change. Re-entry protocols based on evidence and a systematic approach would reduce personal bias and contribute to an engineered seismic management plan that is defensible. This paper investigates the research methodology used to optimise each re-entry assessment system and the results of the comparison. Potential improvements to the analysis techniques and opportunities for further research are discussed at the end of the paper.

\section{$2 \quad$ Background}

Seismic hazard is time dependent and can increase with the occurrence of large events and mining activities, such as production blasting (Bottiglieri et al. 2009). Mine blasting and large events both cause a rapid change in the stress field, along with dynamic stress waves radiating from the source (Orlecka-Sikora et al. 2012). There is an initial swarm of seismic events around the source that gradually decays in frequency towards background levels similar to before the response was triggered. The initial increase in seismic activity tends to be near the source related to the stress change, but there can also be a family of seismic events further from the source. Events not in the immediate vicinity of the source are either uncorrelated with time, or only loosely correlated, and due to broader stress redistributions and movement of major discontinuities.

Multiple authors have claimed reasonable success in the estimation of seismicity near excavations with rigorous calibration of numerical models (Beck \& Brady 2002; Beck et al. 1997; Potvin \& Hudyma 2001), but more remote activity associated with complex geometry and geology has proven much more difficult to replicate. Even when there has been a correlation identified between modelling and seismicity, the resolution is too coarse to benefit re-entry practices on a daily basis.

Re-entry protocols can only be expected to limit the exposure of personnel to large events. The reliance on re-entry methods for risk reduction may vary between sites depending on the overall level of seismic risk and the effectiveness of other seismic management controls.

Disley (2014) used a range of source parameters in the re-entry assessment at Kidd Mine. Parameters including static/dynamic stress drop and apparent stress were visually interpreted to inform the re-entry decision, but the rules were not definitive and most parameters only varied over long time scales.

Vallejos and McKinnon (2009) conducted a survey of 18 seismically active mines to determine the prevailing re-entry practices, mostly in Ontario. A large majority of mines (89\%) developed their re-entry protocols in-house from local experience. This resulted in a wide variety of methods used, probably because there were no widely documented methods available. Over half the mines used event count as the primary parameter for re-entry decisions. The remainder used a form of analysis related to event magnitude or energy, while some mines had blanket rules for a spherical exclusion distance and period of time based on crew comfort levels. Each of these groups were investigated in this research in an endeavour to determine whether any advantages could be found in a particular method. Documented re-entry methods have rarely been supported by systematic back analysis of a large number of cases. This research uses large databases to optimise and compare the success of each method.

\section{Methodology}

\subsection{Definition of seismic responses}

A seismic response is defined as an elevated activity rate over a finite time period and is distinguishable from background seismicity, which is weakly clustered in space and time. The Omori Law (Omori 1894) was 
the first to describe the decreasing frequency of aftershocks following earthquakes. Utsu (1961) proposed the Modified Omori Law (MOL), as per Equation (1). Utsu et al. (1995) reviewed the Omori formula 100 years after it was first documented and summarised its wide acceptance and applicability to seismic responses.

$$
n(t)=\frac{K}{(t+c)^{p}}
$$

where:

$$
\begin{aligned}
& n(t)=\text { Events per interval of time, at time } t . \\
& K \quad=\text { Productivity constant. } \\
& P \quad=\text { Decay constant. } \\
& C \quad=\text { Time offset constant. }
\end{aligned}
$$

A method for estimating the $K, p$ and $c$ parameters of the MOL was provided by Ogata (1983) and uses the maximum likelihood estimation process. Nyffenegger and Frohlich (2000) described the same procedure but highlighted the uncertainties calculated for each parameter were not representative of the overall suitability of fit to the seismic record. It was recommended that the Anderson-Darling statistic (Anderson \& Darling 1954) be included in all determinations of the MOL parameters. Woodward and Wesseloo (2015) studied the identification and delineation of seismic sequences and implemented a method in the mXrap software (Harris \& Wesseloo 2015) to extract the time and location of seismic responses and estimate the $K, p$ and $c$ parameters and the associated Anderson-Darling statistic.

Many re-entry assessments are only conducted for seismic responses to blasting since a blast database is often available for the quick identification of responses. The events are then linked to blasts if they are within a certain distance and time period. For this work, all seismic responses were modelled whether related to a blast, large event or otherwise, using the method developed by Woodward and Wesseloo (2015). This method spatially and temporally delineates seismic responses within a seismic event database. The sequences were modelled with the $\mathrm{MOL}$ and the events that were reliably represented were removed from the database. This allowed further investigation into additional triggers that were more weakly clustered (Woodward \& Wesseloo 2015; Woodward et al. 2017).

A total of 1,604 responses were modelled across three hard rock underground mines in Australia and Canada. The seismic catalogues were first reviewed to ensure there were no major changes in system sensitivity or source parameter calculations in the period analysed (Morkel \& Wesseloo 2017). A summary of the modelled responses for each mine is presented in Table 1 and the distribution of the $K$ and $p$ parameters is plotted in Figures 1 and 2. The $K$ and $p$ parameters can be represented with a log-normal distribution similar to the responses modelled by Vallejos and McKinnon (2009). The $p$ distribution was similar for all three mines, although Mine A had slightly more responses with lower decay rates. Mine B typically had a higher number of events associated with seismic responses; Mine A typically the least. 
Table 1 Summary of modelled responses

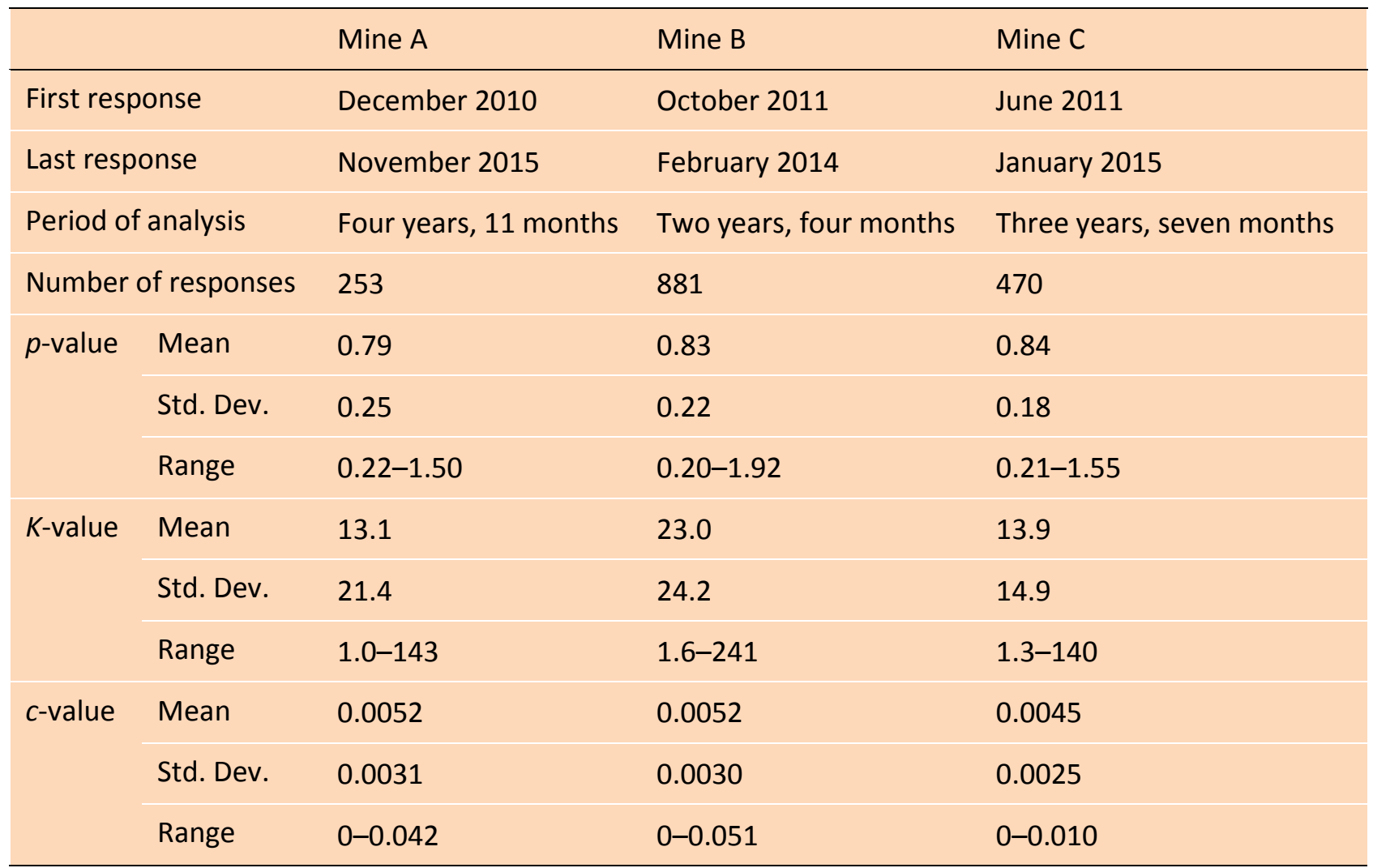

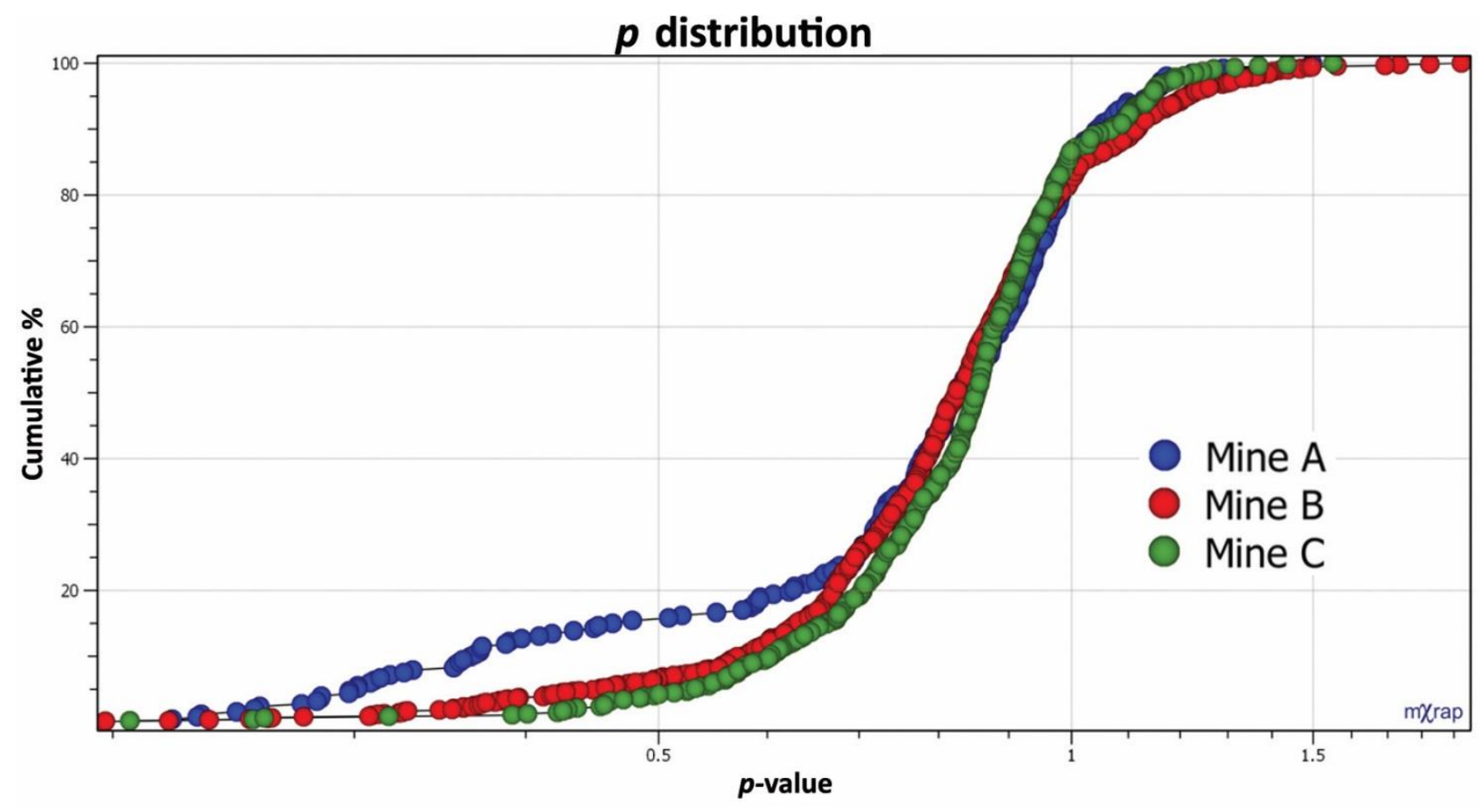

Figure 1 Cumulative distribution of $p$-value (decay parameter) for each mine 


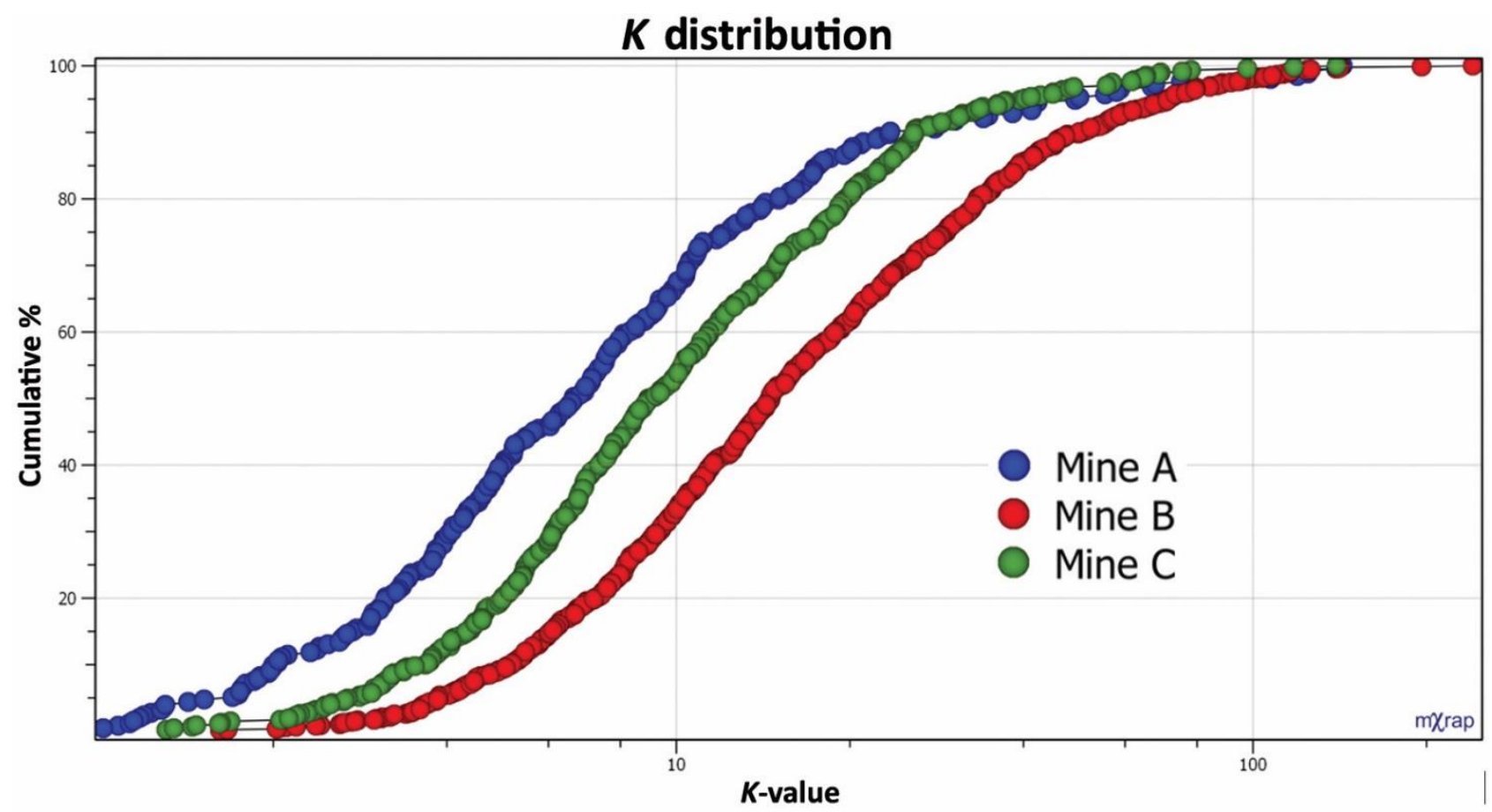

Figure 2 Cumulative distribution of $K$-value (productivity parameter) for each mine

\subsection{Definition of re-entry assessment success}

The success of each re-entry assessment methodology was judged based on two parameters; the average period of exclusion that resulted from the re-entry rules, and the percentage of large events that occurred within the re-entry time. A good re-entry protocol minimises the period of exclusion while maximising the portion of large events within the exclusion. Large events were linked to the modelled responses if they were within $150 \mathrm{~m}$ and $72 \mathrm{hrs}$ of the start of the response. Each large event was linked to the closest response which occurred in the 72 hrs prior. Large events within 15 mins of the response initiation were not included in the back analysis. Those events do not give an indication of a successful re-entry method since almost any rule would prevent exposure to these events.

The local magnitude $\left(\mathrm{M}_{\mathrm{L}}\right)$ that defined a large event for back analysis was adjusted, as per Table 2. Even though these magnitudes are not always considered hazardous, increasing the magnitude threshold reduces the size of the dataset. Smaller datasets make conclusions less reliable, therefore, more moderate sized events were used. The definition of local magnitude can also vary between sites and can be based on seismic moment, energy or a combination. The occurrence of moderate magnitude events should be related to large events, as per the frequency-magnitude relationship.

Table 2 Back analysis event local magnitudes and corresponding size of the dataset for each mine

\begin{tabular}{llll}
\hline Number of events & Mine A & Mine B & Mine C \\
\hline$>+1.0 \mathrm{ML}_{\mathrm{L}}$ & 20 & 12 & 4 \\
$>+0.5 \mathrm{M}_{\mathrm{L}}$ & 56 & 49 & 20 \\
$>0.0 \mathrm{M}_{\mathrm{L}}$ & 148 & 186 & 56 \\
$>-0.5 \mathrm{M}_{\mathrm{L}}$ & 400 & 698 & 244 \\
\hline
\end{tabular}

A re-entry protocol that assesses events in real-time should also be more successful than blanket rules, where a fixed exclusion is defined for all responses irrespective of the observed activity. This is because real-time methods require additional work in implementation and add uncertainty to mine scheduling and planning. This concept is depicted in Figure 3 where the success of a blanket re-entry rule is plotted. This 
highlights the strong correlation of large events with responses and how a large portion of events can be captured by relatively short exclusions. Progressively longer exclusions are required for each percentage increase in captured events.

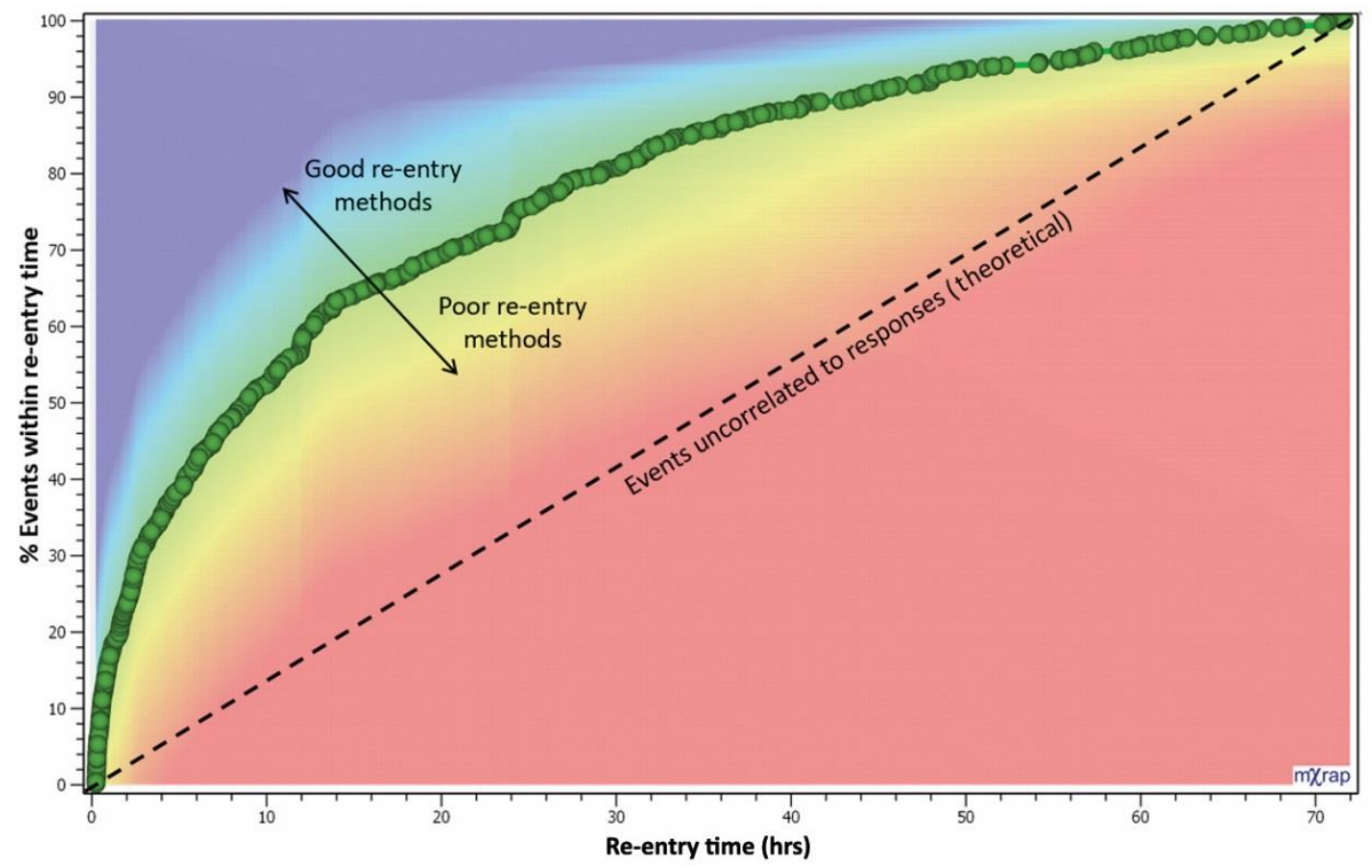

Figure 3 Blanket re-entry rule success for events $\geq-0.5 \mathrm{M}_{\mathrm{L}}$ at Mine $\mathrm{B}$. The blanket rule is a good basis for comparison to real-time assessment techniques

\subsection{Vallejos and McKinnon method}

Vallejos and McKinnon (2009) assessed 340 seismic responses to blasting from nine seismically active mines, mostly in Ontario, and proposed a methodology for re-entry analysis based on three different protocols that consider the rate of seismic events compared to historical behaviour. Morkel and Rossi Rivera (2017) investigated the practical use of the Vallejos and McKinnon method and implemented the approach in $m X r a p$ (Harris \& Wesseloo 2015), that requires the specification of three threshold parameters that all need to be met before re-entry is permitted.

\subsubsection{Protocol 1 - seismic envelopes}

The Vallejos and McKinnon method assumes a log-normal distribution in the $p$ and $K$ parameters to obtain a series of seismic envelopes from a Monte Carlo simulation of the cumulative version of the MOL (Vallejos \& McKinnon 2010). The simulation is only valid from one hour after the start of the response. Responses with $c$ parameters higher than 0.1 or Anderson-Darling statistics above 2.0 were not considered in the simulation.

These seismic envelopes are shown in Figure 4 with an example response from Mine A, along with the interpolated percentile value for each event. The percentile of the envelope represents the probability that the response will be below that level. Vallejos and McKinnon (2009) suggested that re-entry should only occur when the cumulative event count crosses the seismic envelopes sub-horizontally. Morkel and Rossi Rivera (2017) interpreted this as a percentile drop.

The first protocol is a required percentile drop from the previous maximum. Since the seismic envelopes cluster tightly at the start of the second hour, this protocol is only applied 30 minutes after the envelopes start. This means the Vallejos and McKinnon method has a minimum exclusion of 90 mins. 


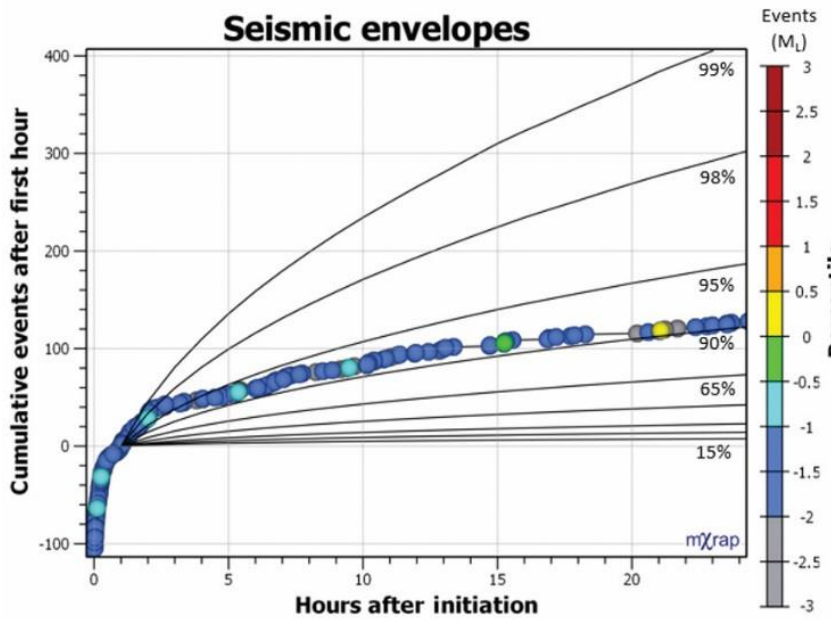

(a)

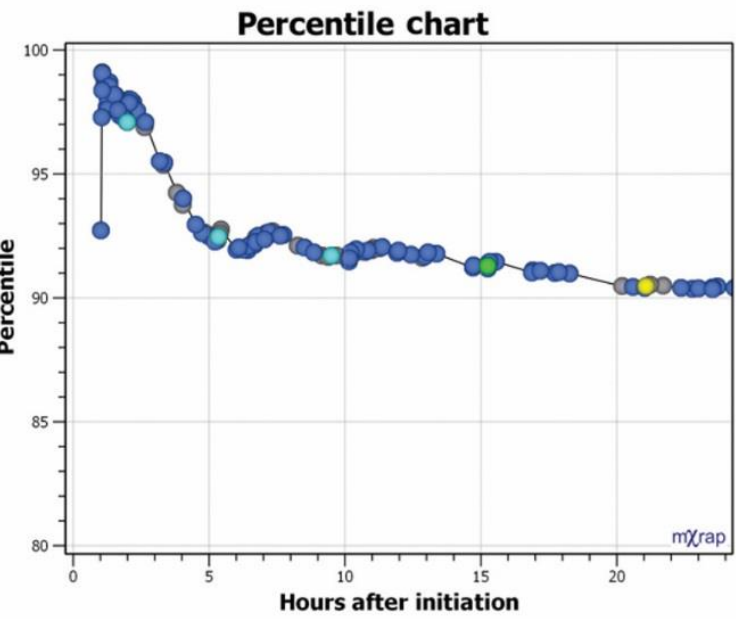

(b)

Figure 4 Example response from Mine A depicted as a: (a) seismic envelopes plot; and, (b) related percentile chart

\subsubsection{Protocol 2 - time of maximum curvature ( $\left.T_{M C}\right)$}

Vallejos and McKinnon (2009) included the calculation of the time of maximum curvature ( $T_{M C}$, Equation (2)) for each iteration of the Monte Carlo simulation, as this value represents the transition between a high and low event rate and is thus suitable for re-entry. The $T_{M C}$ percentile boundaries shown in Figure 5 represent the probability that the current response has not yet reached the time of maximum curvature. Morkel and Rossi-Rivera (2017) eliminated the need for the $K$ parameter in the $T_{M C}$ calculation and, therefore, the need for a Monte Carlo simulation by expressing Equation (2) in terms of $K$ and substituting into the MOL (Equation (3)). The second protocol is that the hourly event rate must fall below the specified $T_{M C}$ boundary.

$$
\begin{gathered}
T_{M C}=\left[K p \sqrt{\frac{2 p+1}{p+2}}\right]^{\frac{1}{p+1}}-c \\
n\left(T_{M C}\right)=\frac{T_{M C}}{p \sqrt{\frac{2 p+1}{p+2}}}
\end{gathered}
$$

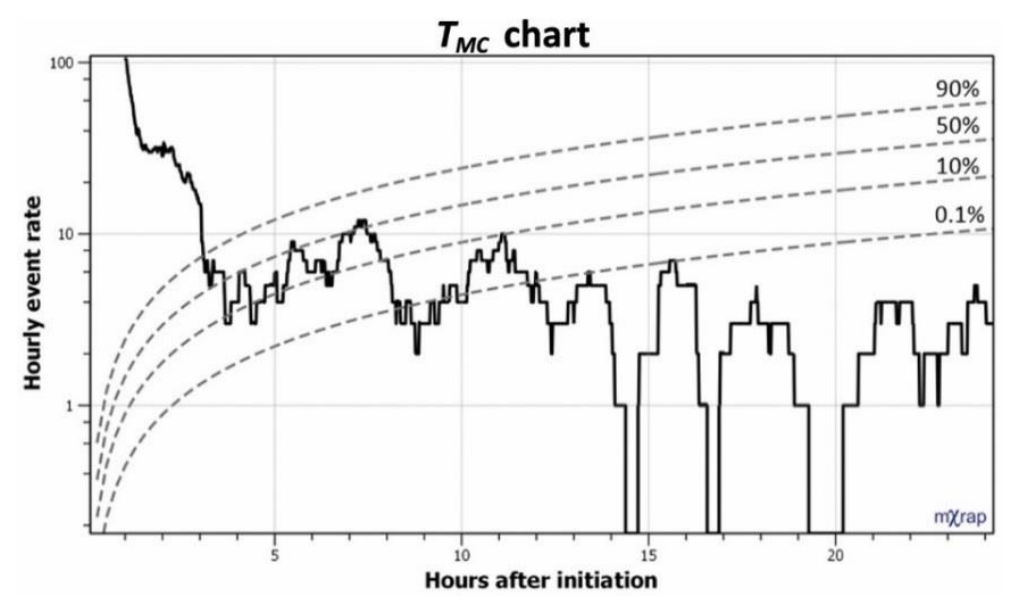

Figure $5 \quad T_{M C}$ chart for the same response as Figure 4 


\subsubsection{Protocol 3 - background level}

The third protocol specifies that the ratio between the current event rate and the background rate must fall below a specified value. Similar to the $T_{M C}$ analysis, the event rate is calculated with a moving time window of one hour and, therefore, cannot be calculated for the first hour after the response is initiated.

In this implementation, the background rate was calculated for each response from the cumulative ascending distribution of the diurnal chart for events within the previous six months and within a $150 \mathrm{~m}$ spherical radius. Only unmodelled events were used to reduce the influence of blasting and large events. The eleventh highest hourly bin (adjusted for the time span) was used as the background value (Figure 6).

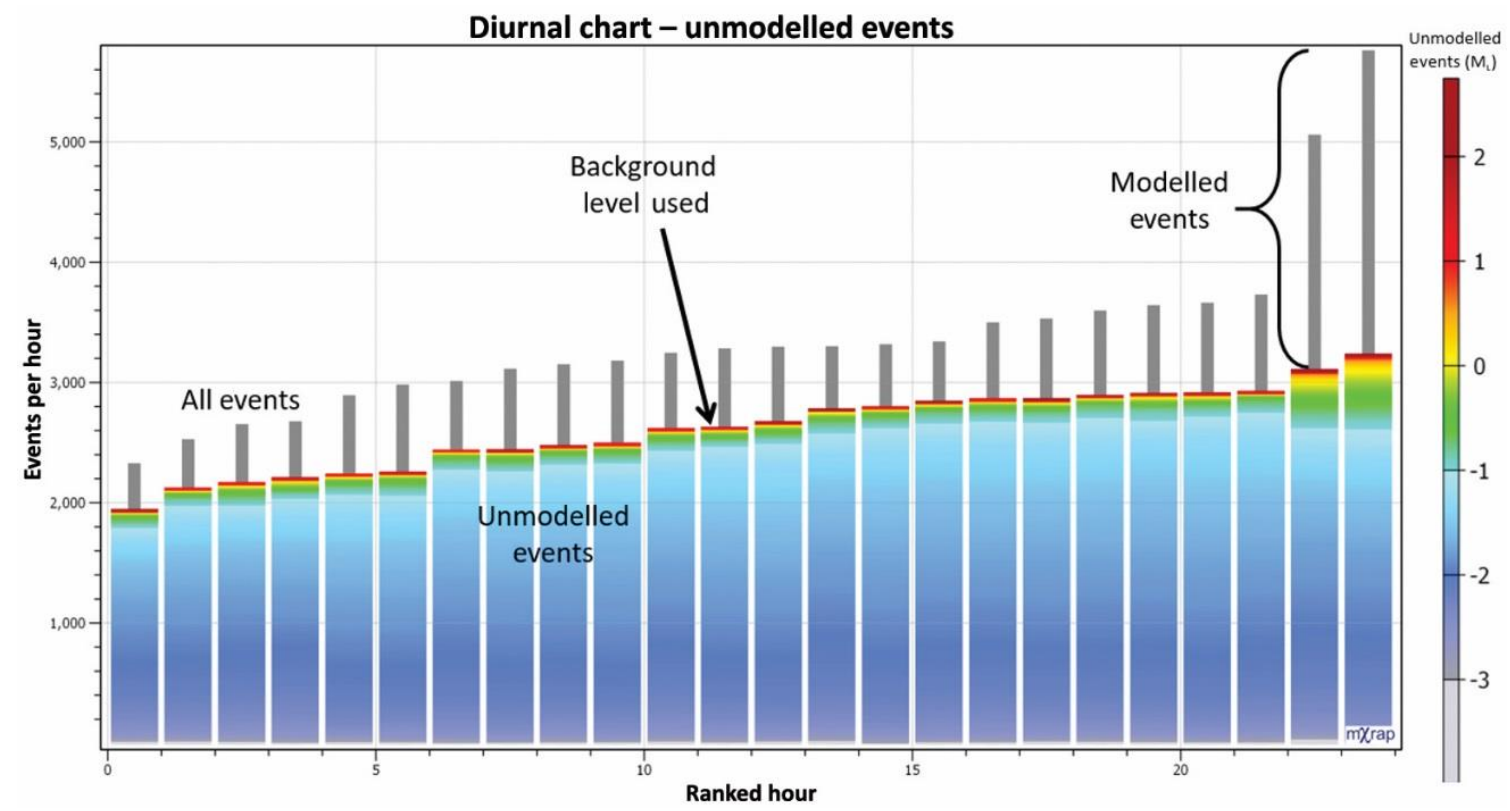

Figure 6 Diurnal chart from Mine A over the analysis period. Hours are ranked by the number of events

\subsubsection{Vallejos and McKinnon optimisation}

The Vallejos and McKinnon method requires that each of the three thresholds is met before re-entry is permitted. Some responses were found to not meet the re-entry guidelines and, therefore, a maximum re-entry time needed to be defined. This was set at $72 \mathrm{hrs}$ to align with the back analysis time span.

The variation in success resulting from different threshold values was investigated through a brute force approach. Each combination of the threshold values in Table 3 were applied to all defined responses at each of the three mines. For each combination, the average time of the exclusion was calculated along with the number of large events that were captured.

Table 3 Vallejos and McKinnon optimisation parameters

\begin{tabular}{llll}
\hline & Minimum & Maximum & Number of iterations \\
\hline Percentile drop & $0.1 \%$ & $40 \%$ & 29 \\
$T_{M C}$ percentile & $0.01 \%$ & $99.9 \%$ & 62 \\
Background ratio & 0.01 & 40 & 34 \\
\hline
\end{tabular}

Combination of thresholds that result in the same success but had longer exclusions than other combinations were then removed to arrive at the optimised Vallejos and McKinnon results. Figure 7 shows this process for events greater than $0.0 \mathrm{M}_{\mathrm{L}}$ at Mine $\mathrm{C}$. It was rare for the Vallejos and McKinnon method to arrive at re-entry times less than three hours or longer than two days. It was noted during the analysis that the percentile drop threshold (first protocol) had the highest influence on the resulting re-entry time and 
the subsequent success. The other two thresholds could be a much wider range while not influencing the results.

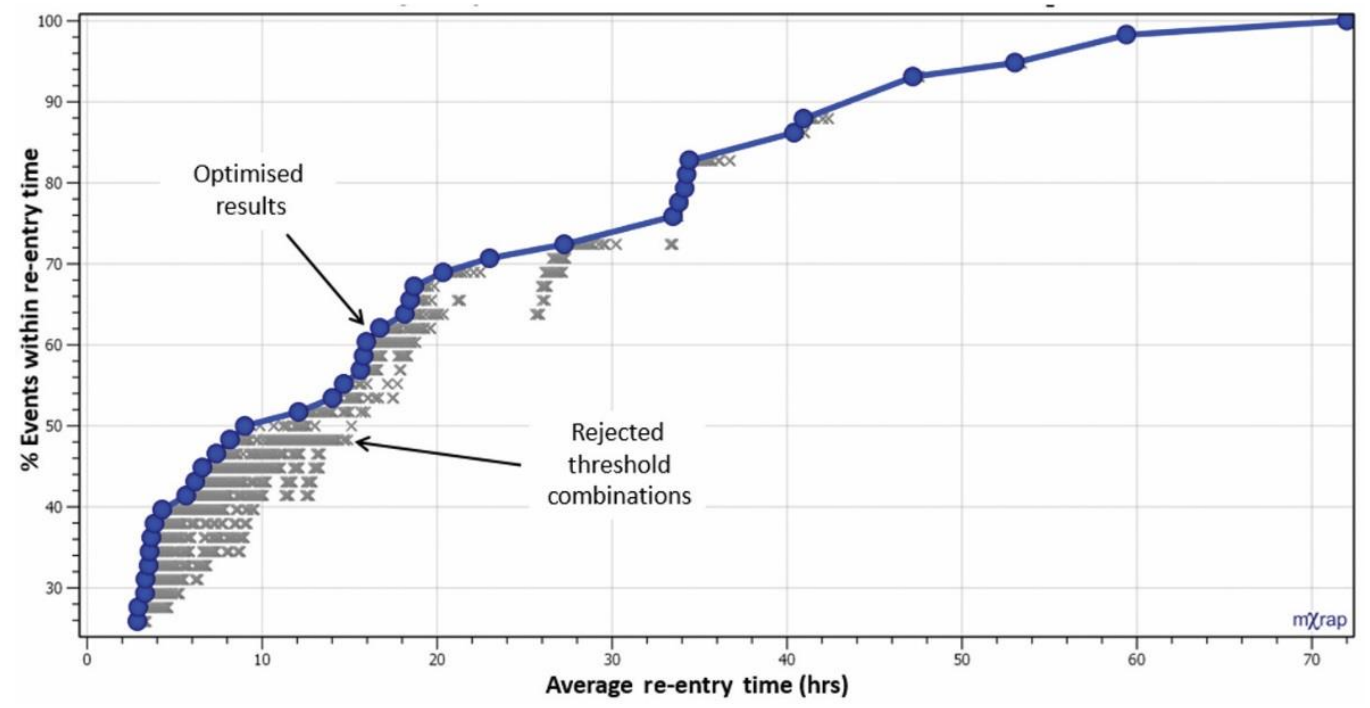

Figure 7 Vallejos and McKinnon brute force optimisation for $0.0 \mathrm{M}_{\mathrm{L}}$ events at Mine $\mathrm{C}$

\subsection{Energy and event based optimisation}

Penney and Hills (2013) reviewed re-entry and exclusion practices at the Tasmania Mine and established seismic parameter thresholds that must be met prior to re-entry. This approach reviewed the impact for production of implementing these rules from back analysis but did not consider whether the rules resulted in improved risk management. The rules included a micro threshold to reset the exclusion clock, if exceeded, based on the background energy release rate and event rate. A macro threshold was also defined to initiate an exclusion when a certain magnitude event occurred.

Alcott et al. (1998) presented a technique for assessing rockburst hazard using three seismic parameters to categorise events into source mechanisms and identify precursory trends that indicate worsening or improving conditions over time. Precursory trends, if identified, are important to consider in re-entry assessments since, by definition, they indicate an increased short term hazard. Seismic energy, apparent stress and seismic moment were the parameters used to classify seismic source mechanisms, and energy was used to identify precursory trends to large events.

Case studies using the method resulted in occasional precursory trends being highlighted but Alcott et al. (1998) concluded the system was more useful to identify seismic sources and long term hazard than for short term hazard assessment. The precursory trends were not quantified but a simple approach was adopted for this study, loosely based on Alcott et al. (1998), that defines re-entry when the seismic energy released over a moving time window drops below a specified value. This approach has two main input parameters that require optimisation to minimise the average exclusion period, while maximising the number of large events that occur within the exclusion period.

A similar approach was also taken that merely uses the number of events in the moving time window as the re-entry protocol. This will test whether the size of the events has any influence on the short term hazard. The same optimisation process was undertaken as the Vallejos and McKinnon method, with the brute force parameters summarised in Table 4. The minimum possible re-entry is the length of the moving time window. The time window was found to have a strong influence on the resulting re-entry time and the number of large events captured by both of these re-entry rules. 
Table 4 Brute force optimisation parameters for energy and event based methods

\begin{tabular}{lllll}
\hline & Minimum & Maximum & Increment & Number of iterations \\
\hline $\log _{10}$ (Energy) & 0.05 & 5.0 & 0.05 & 100 \\
Event count & 1 & 300 & 1 & 300 \\
Moving time window & $0.1 \mathrm{hrs}$ & $24 \mathrm{hrs}$ & $0.1 \mathrm{hrs}$ & 240 \\
\hline
\end{tabular}

\section{$4 \quad$ Results}

The results of each optimisation were collected to assess the relative success of each of the four re-entry methods. Figure 8 shows an example of the results of each re-entry method for events over $-0.5 \mathrm{M} L$ at Mine A. There are clear differences in the re-entry method performance and there are occasionally several hours longer exclusions for the same number of large events captured.

The energy and event based methods both outperformed the blanket re-entry rule but the Vallejos and McKinnon method was less efficient. The difference between the methods is best highlighted by the variation in captured events for constant re-entry time or vice versa. Figure 9 shows the difference in average re-entry time compared with the blanket rule for the same case as Figure 8.

The results from each mine are summarised in Table 5 for all four back analysis magnitudes. The average difference in re-entry times compared with the blanket rule are coloured from 'cold' to 'hot', in order from shortest to longest exclusion time. The energy method was the best method in the majority of cases, outperforming the similar event count method in all but one case. Both of these consistently outperformed the blanket rule. The Vallejos and McKinnon method was regularly the worst performed and was less efficient then the blanket rule in the majority of cases.

The Vallejos and McKinnon method performed worse in Mine A than for Mines B and C. This method also typically performed better for back analysis magnitudes +0.5 and $+1.0 \mathrm{M}_{\mathrm{L}}$, but this is less reliable given the small number of events in the dataset that are above these magnitudes.

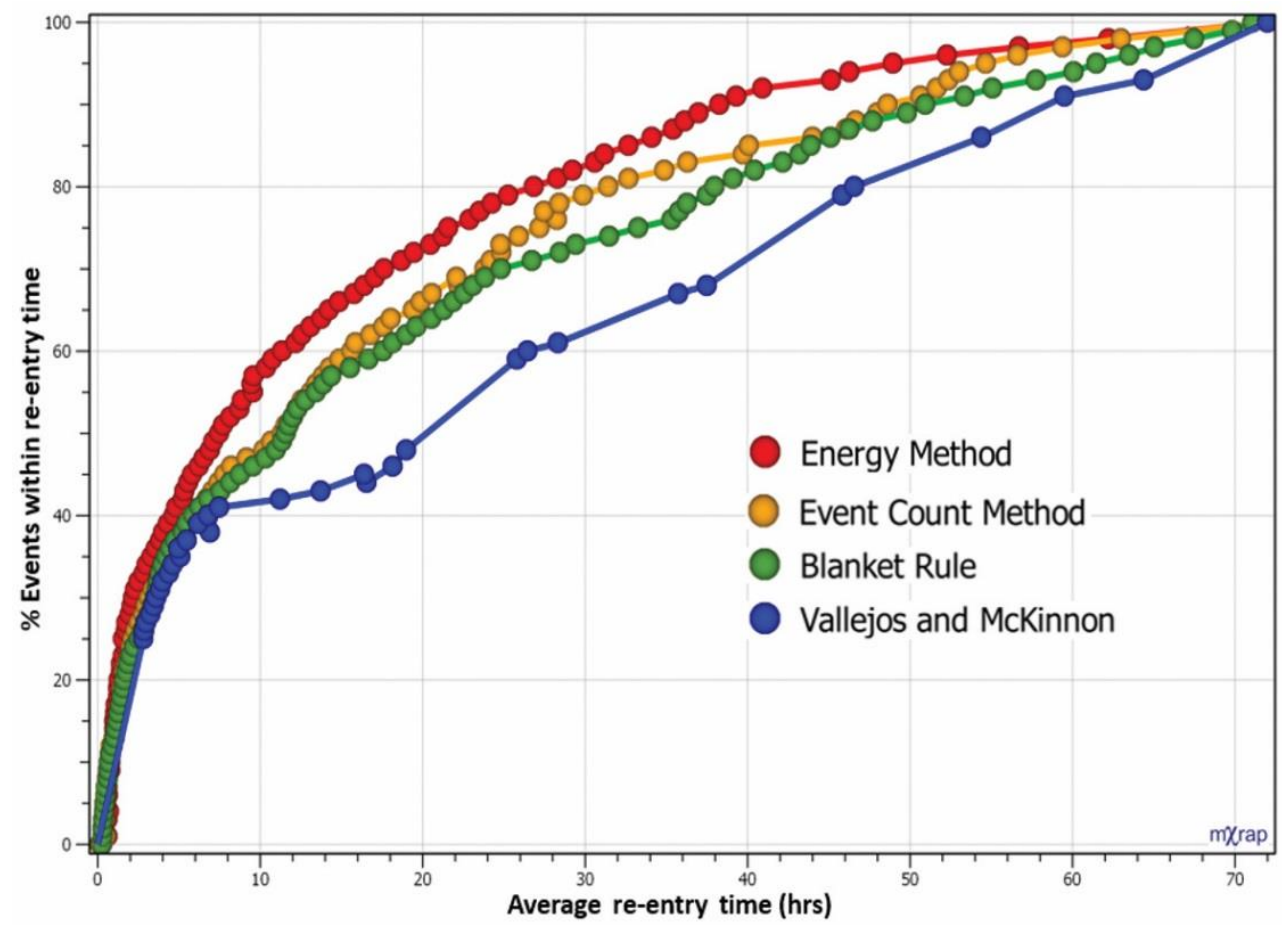

Figure 8 Comparison of the relative success of each re-entry assessment method for $-0.5 \mathrm{M}_{\mathrm{L}}$ events at Mine A 


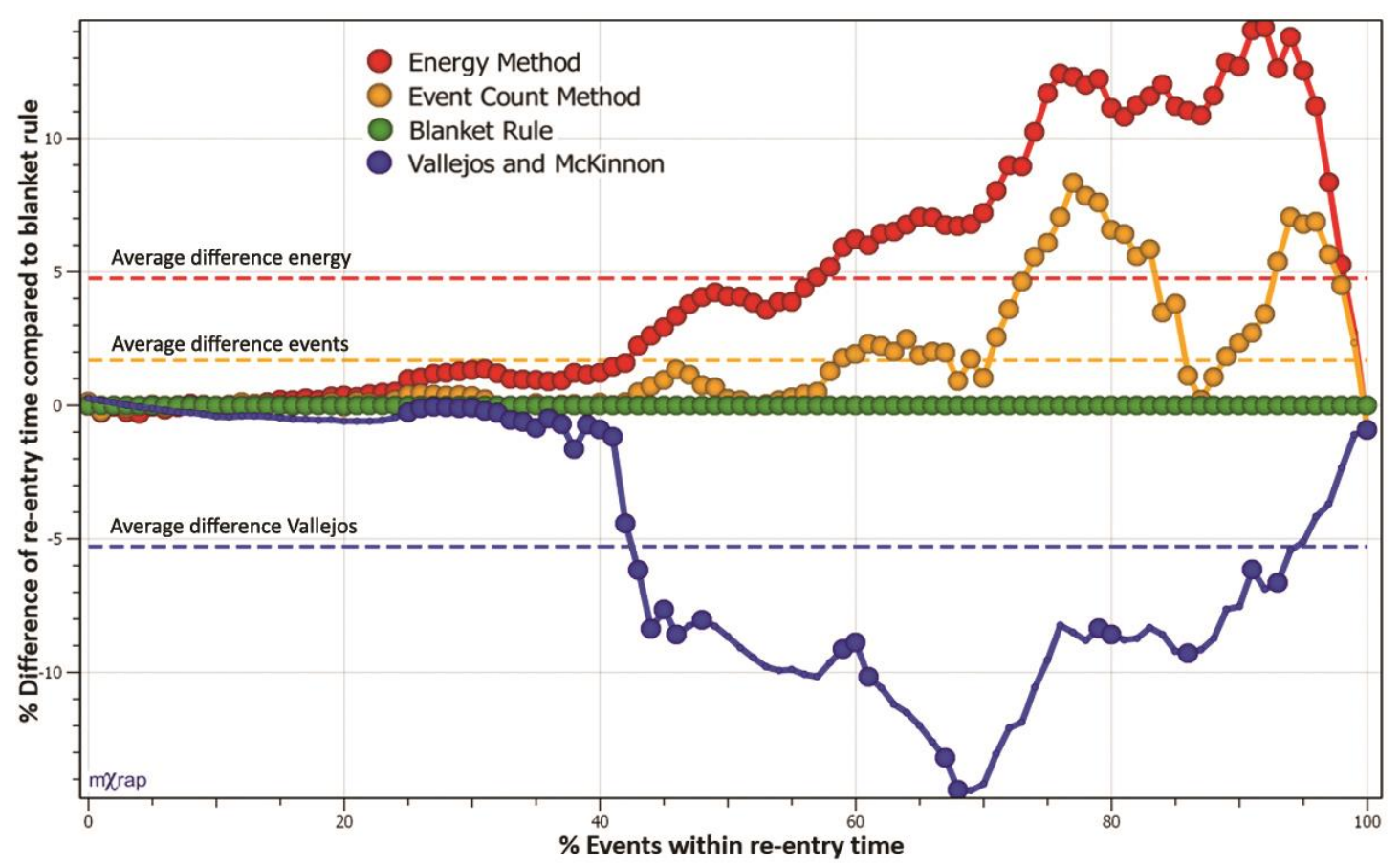

Figure 9 Comparison of re-entry assessment methods to the blanket rule for events over $-0.5 \mathrm{M}_{\mathrm{L}}$ at Mine A. Average difference for each real-time method is also plotted (dotted lines)

Table 5 Summary of results from all three mines and various back analysis magnitudes. The average difference in re-entry time compared with the blanket rule are coloured from 'cold' to 'hot' for the shortest to longest exclusion time in each case

\begin{tabular}{l|l|l|l|l|l|} 
& & \multicolumn{5}{c}{ Average difference in re-entry time compared to blanket rule (hrs) } \\
\hline Mine & $\begin{array}{l}\text { Back analysis } \\
\mathrm{M}_{\mathrm{L}}\end{array}$ & Energy method & $\begin{array}{l}\text { Event count } \\
\text { method }\end{array}$ & Blanket rule & $\begin{array}{l}\text { Vallejos and } \\
\text { McKinnon }\end{array}$ \\
\hline A & $+1.0 \mathrm{M}_{\mathrm{L}}$ & +5.6 & +3.5 & 0.0 & -2.2 \\
\hline A & $+0.5 \mathrm{M}_{\mathrm{L}}$ & +5.0 & +1.8 & 0.0 & -4.5 \\
\hline A & $0.0 \mathrm{M}_{\mathrm{L}}$ & +5.0 & +1.8 & 0.0 & -5.6 \\
\hline A & $-0.5 \mathrm{M}_{\mathrm{L}}$ & +4.8 & +1.7 & 0.0 & -5.3 \\
\hline B & $+1.0 \mathrm{M}_{\mathrm{L}}$ & +11.6 & +10.2 & 0.0 & +2.8 \\
\hline B & $+0.5 \mathrm{M}_{\mathrm{L}}$ & +6.6 & +6.4 & 0.0 & +4.1 \\
\hline B & $0.0 \mathrm{M}_{\mathrm{L}}$ & +5.3 & +5.2 & 0.0 & -0.6 \\
\hline B & $-0.5 \mathrm{M}_{\mathrm{L}}$ & +4.6 & +4.7 & 0.0 & -1.2 \\
\hline C & $+1.0 \mathrm{M}_{\mathrm{L}}$ & +3.7 & +3.3 & 0.0 & -0.3 \\
\hline C & $+0.5 \mathrm{M}_{\mathrm{L}}$ & +5.8 & +5.3 & 0.0 & +3.7 \\
\hline C & $0.0 \mathrm{M}_{\mathrm{L}}$ & +6.9 & +6.6 & 0.0 & +3.8 \\
\hline C & $-0.5 \mathrm{M}_{\mathrm{L}}$ & +3.9 & +3.5 & 0.0 & -2.1 \\
\hline
\end{tabular}




\section{Discussion}

This research differed from many other re-entry assessments by using all responses, as opposed to just blasting related responses. This removes the need for a detailed blast database that is not available at all sites. The additional responses are also useful for sites with a more limited history of seismic activity. The modelling of each response has the benefit of removing delayed responses from the analysis, which means that longer analysis times are possible. A minimum of $72 \mathrm{hrs}$ was used for each response, where blasting responses are typically limited to 24 hrs to remove the effects of the next day's blasting. Unfortunately, there is some bias in the definition of the response since the database is visually scanned for responses to model. While every effort was made to be consistent, an automated approach would ensure a clear distinction between a modelled response and a brief, spurious spike in activity that is not included. An automated approach to response modelling would also make it more likely that this type of re-entry assessment is undertaken at site level since the manual process can be time consuming for large databases.

The triggering event for each response, e.g. blast or large event, was not investigated. Further research into the response triggers would allow for more practical rules for exclusions after different sized blasts or events. For this study, it was assumed that an exclusion began at the start of each response and was not lifted until the re-entry rules were met. If there were more rules governing the initiation of the exclusion there could potentially be more efficient results. No consideration was given to the size of the excluded area for each response. Presumably each site would consider the excluded area case-by-case, depending on the specific mine geometry and known seismic sources.

A relatively simple method of back analysis was used to gauge the success of each re-entry assessment methodology but some of the parameters were quite arbitrarily defined. A large event was linked to a response if it occurred within $150 \mathrm{~m}$ and 72 hrs of the initiation. Clearly, the success percentages vary depending on this time period of analysis and there may be some large events that were linked to an unrelated rock mass response. Particularly for the Vallejos and McKinnon method, the results might improve if the length of the back analysis time window was decreased to $24 \mathrm{hrs}$.

The common problem with large event back analysis is the limited size of the dataset to generate results. This can make conclusions more uncertain. It was necessary to investigate the occurrence of moderate size events and assume the behaviour of their occurrence can be a proxy for large event behaviour. In cases where the frequency-magnitude relationship follows a power law this would be a reasonable assumption. Further research may investigate alternate methods of quantifying the success of re-entry methods, such as comparing the frequency-magnitude distribution of events within and outside of exclusion. This may offer a better assessment of the difference in hazard before and after re-entry for each of the assessment methods.

It is always important to consider the quality of the seismic data when conducting this sort of analysis, particularly when counting the number of events. The sensitivity of the seismic system will affect the number of recorded events and, therefore, the $K$ parameter and the optimum threshold for the activity rate prior to re-entry. The definition and consistency of the recording of energy and local magnitude may also vary between sites (Morkel \& Wesseloo 2017; Morkel et al. 2015), this is why responses were not combined for the three mines assessed. The seismic data was reviewed prior to modelling responses to ensure the time period of assessment had no major variations in the system sensitivity or source parameter calculations, but there were no additional quality filters applied to the data. This was an attempt to maximise the data available for investigation. If further research aims at finding specific re-entry rules for a mine, or to find generic re-entry rules between sites, much more care will be needed to account for data quality variations in space and time. This research could help mines to define re-entry protocols that do not have a large seismic database to conduct their own back analysis.

There were some assumptions required to systematically implement the Vallejos and McKinnon method. The percentile drop threshold (first protocol) was the dominant factor in the re-entry time and was the most common reason for a re-entry time to be capped at the maximum $72 \mathrm{hrs}$. This is because responses that begin in the very high percentiles for the first $2-3 \mathrm{hrs}$, e.g. $99 \%$, will take a long time to drop by $5-10 \%$, 
even if there is very little subsequent recorded activity. This is very different to responses that begin in the middle envelopes where the percentage can drop much more quickly. Perhaps a different methodology can be implemented to account for this effect in future research but it may also be beneficial that very high responses will not quickly reach the re-entry thresholds. This protocol was not precisely defined by Vallejos and McKinnon (2009). The effects of permitting re-entry when only two of the three protocols are met may also be investigated but this would be against the original procedure described by Vallejos and McKinnon (2009).

The energy and event based methods have much less room for ambiguity in their implementation since each has only two key parameters. For both methods, the moving time window was the dominant parameter influencing the success of the re-entry assessment. Given there was no variation in the time window used in the Vallejos and McKinnon method, and the dominance of this parameter in the energy and event based methods, it is possible that better results could be obtained with a variable time window in the Vallejos and McKinnon method. A one hour time window was used in the event rates that were inputs for the second and third protocols of the Vallejos and McKinnon method.

\section{Conclusion}

The results of this research indicate that a real-time re-entry assessment method can offer improved outcomes, compared to blanket re-entry rules, by reducing the average exclusion time while still capturing the same number, or a higher number, of large events. The incorporation of event size in the assessment can result in better results than the event count.

The Vallejos and McKinnon method is a probabilistic framework for re-entry assessment but this was found to be less efficient. That is, for the same re-entry time less events are captured than the blanket rule in the majority of cases in this study.

Several potential improvements to the analysis techniques and avenues for further research have been discussed. The tools developed in this research can be used onsite to inform specific decision-making and provide a justification for the re-entry protocols that are implemented.

\section{Acknowledgement}

The authors thank Johan Wesseloo and the mXrap team at the Australian Centre for Geomechanics for assistance during this project. We also thank the mXrap Consortium for providing the $\mathrm{mXrap}$ software and seismic databases. Dr Kyle Woodward assisted with the identification and delineation of seismic responses. This research is part of a Master of Engineering Science (Mining Geomechanics) at Curtin University undertaken by Stuart Tierney, supervised by Mostafa Sharifzadeh.

\section{References}

Alcott, JM, Kaiser, PK \& Simser, BP 1998, 'Use of microseismic source parameters for rockburst hazard assessment', Pure and Applied Geophysics, vol. 153, pp. 41-65.

Anderson, TW \& Darling, DA 1954, 'A test of goodness of fit', Journal of the American Statistical Association, vol. 49, no. 268, pp. 765-769.

Beck, DA \& Brady, BHG 2002, 'Evaluation and application of controlling parameters for seismic events in hard-rock mines', International Journal of Rock Mechanics and Mining Sciences, vol. 39, pp. 633-642.

Beck, DA, Brady, BHG \& Grant, D 1997, 'Induced stress and microseismicity in the 3000 orebody, Mount Isa', Geotechnical and Geological Engineering, vol. 15, pp. 221-233.

Bottiglieri, M, Lippiello, E, Godano, C \& de Arcangelis, L 2009, 'Identification and spatiotemporal organization of aftershocks', Journal of Geophysical Research: Solid Earth, vol. 114, no. B3, pp. 1-12.

Cook, NGW 1976, 'Seismicity associated with mining', Engineering Geology, vol. 10, pp. 99-122.

Disley, NV 2014, 'Seismic risk and hazard management at Kidd Mine', in M Hudyma \& Y Potvin (eds), Proceedings of The Seventh International Conference on Deep and High Stress Mining, 16-18 September 2014, Sudbury, Australian Centre for Geomechanics, Perth, pp. 107-122.

Hadjigeorgiou, J \& Potvin, Y 2011, 'A critical assessment of dynamic rock reinforcement and support testing facilities', Rock Mechanics and Rock Engineering, vol. 44, no. 5, pp. 565-578. 
Harris, PC \& Wesseloo, J 2015, mXrap, version 5, Australian Centre for Geomechanics, Perth, www.mXrap.com

Kahneman, D 2003, 'A perspetive on judgement and choice - mapping bounded rationality', American Psychologist, vol. 58, no. 9, pp. 697-720.

Mendecki, A 2008, 'Forecasting seismic hazard in mines', in Y Potvin, J Carter, A Dyskin \& R Jeffery (eds), Proceedings of The First Southern Hemisphere International Rock Mechanics Symposium, vol. 1, 16-19 September 2008, Perth, Australian Centre for Geomechanics, Perth, pp. 55-71.

Morkel, IG \& Rossi-Rivera, P 2017, 'The implementation and quantification of the Vallejos and McKinnon re-entry methodology', in J Wesseloo (ed.), Proceedings of The Eighth International Conference on Deep and High Stress Mining, 28-30 March 2017, Perth, Australian Centre for Geomechanics, Perth, in print.

Morkel, IG \& Wesseloo, J 2017, 'A method to determine systematic shifts in micro-seismic databases', in J Wesseloo (ed.), Proceedings of The Eighth International Conference on Deep and High Stress Mining, 28-30 March 2017, Perth, Australian Centre for Geomechanics, Perth, in Print.

Morkel, IG, Wesseloo, J \& Harris, PC 2015, 'Highlighting and quantifying seismic data quality concerns', in PM Dight (ed.), Proceedings of The Ninth International Symposium on Field Measurements in Geomechanics, 9-11 September 2015, Sydney, Australian Centre for Geomechanics, Perth, pp. 539-549.

Nyffenegger, P \& Frohlich, C 2000, 'Aftershock occurrence rate decay properties for intermediate and deep earthquake sequences', Geophysical Research Letters, vol. 27, no. 8, pp. 1215-1218.

Ogata, Y 1983, 'Estimation of the parameters in the Modified Omori Formula for aftershock frequencies by the Maximum Likelihood Procedure', Journal of Physics of the Earth, vol. 31, no. 2, pp. 115-124.

Omori, F 1894, 'On the after-shocks of earthquakes', The Journal of the College of Science, vol. 7, Imperial University of Tokyo, Tokyo, pp. 111-200.

Orlecka-Sikora, B, Lasocki, S, Lizurek, G \& Rudziński, $Ł 2012$, 'Response of seismic activity in mines to the stress changes due to mining induced strong seismic events', International Journal of Rock Mechanics and Mining Sciences, vol. 53, pp. 151-158.

Penney, AR \& Hills, PB 2013, 'Development of seismic heading re-entry and exclusion zones at the Tasmania mine', in A Malovichko \& D Malovichko (eds), Proceedings of The 8th International Symposium on Rockbursts and Seismicity in Mines (RaSiM8), Saint Petersburg and Moscow, Geophysical Survey of Russian Academy of Sciences, Obninsk, Mining Institute of Ural Branch of Russian Academy of Sciences, Perm, pp. 447-459.

Potvin, Y 2009, 'Strategies and tactics to control seismic risks in mines', Journal of the Southern African Institute of Mining and Metallurgy, vol. 109, no. 3.

Potvin, Y \& Hudyma, MR 2001, 'Seismic monitoring in highly mechanized hardrock mines in Canada and Australia', in G van Aswegen, R Durrheim \& D Ortlepp (eds), Proceedings of The Fifth International Symposium on Rockburst and Seismicity in Mines 2001, Johannesburg, Southern African Institute of Mining and Metallurgy, Johannesburg, pp. 267-280.

Utsu, T 1961, 'A statistical study of the occurrence of aftershocks', Geophysical Magazine, vol. 30, no. 4, pp. 521-605.

Utsu, T, Ogata, Y \& Matsu'ura, RS 1995, 'A centenary of the omoro formula for a decay law of aftershock activity', Journal of Physics of the Earth, vol. 43, pp. 1-33.

Vallejos, JA \& McKinnon, SD 2010, 'Temporal evolution of afterschock sequences for re-entry protocol development in seismically active mines', in M van Sint Jan \& Y Potvin (eds), Proceedings of The 5th International Seminar on Deep and High Stress Mining, 6-8 October 2010, Santiago, Australian Centre for Geomechanics, Perth, pp. 199-214.

Vallejos, JA \& McKinnon, SM 2009, 'Re-entry protocols for seismically active mines using statistical analysis of aftershock sequences', in M Diederichs \& G Grasselli (eds), The 3rd CANUS Rock Mechanics Symposium, May 2009, Toronto, pp. 1-12.

Woodward, K \& Wesseloo, J 2015, 'Observed spatial and temporal behaviour of seismic rock mass response to blasting', The Journal of the Southern African Institute of Mining and Metallurgy, vol. 115, no. 11, pp. 1044-1056.

Woodward, K, Wesseloo, J \& Potvin, Y 2017, 'The spatial and temporal assesment of clustered and time-dependent seismic responses to mining', in J Wesseloo (ed.), Proceedings of The Eighth International Conference on Deep and High Stress Mining, 28-30 March 2017, Perth, Australian Centre for Geomechanics, Perth, in print. 